\title{
On one family of first passage times of a Markov random walk described by an autoregressive process $\mathrm{AR}(1)$ for nonlinear boundaries
}

\author{
F.G. Rahimov ${ }^{a}$, I.A. Ibadova ${ }^{b}$, L.V. Guliyeva ${ }^{a}$ \\ ${ }^{a}$ Baku State University, Baku, Azerbaijan \\ ${ }^{b}$ Institute of Mathematics and Mechanics of the Azerbaijan National Academy of Sciences, Baku, Azerbaijan
}

\begin{tabular}{|c|c|}
\hline A R T I C L E I N F O & A B S T R A C T \\
\hline $\begin{array}{l}\text { Article history: } \\
\text { Received 28.09.2021 } \\
\text { Received in revised form } 12.10 .2021 \\
\text { Accepted } 18.10 .2021 \\
\text { Available online } 29.12 .2021\end{array}$ & $\begin{array}{l}\text { The consider one family of first passage times of a Markov } \\
\text { random walk described by a first-order autoregressive process } \\
\text { AR(1) for nonlinear boundaries. Limit theorems are proved for a } \\
\text { Markov random walk and a family of first passage times of this } \\
\text { walkfor nonlinear boundaries. }\end{array}$ \\
\hline $\begin{array}{l}\text { Keywords: } \\
\text { First-order autoregressive process } \\
\text { Markov random walk } \\
\text { Central limit theorem } \\
\text { First passage time for a nonlinear } \\
\text { boundary }\end{array}$ & \\
\hline
\end{tabular}

\section{Introduction}

Suppose that $\xi_{n}, n \geq 1$ is a sequence of independent identically distributed random variables defined on some probability space $(\Omega, \mathcal{F}, P)$. As is known, the first-order autoregressive process $(A R(1))$ is determined using a recurrence relation of the form

$$
X_{n}=\beta X_{n-1}+\xi_{n}, \quad n \geq 1,
$$

where $\beta \in R=(-\infty,+\infty)$ is some fixed number and it is assumed that the initial value of $X_{0}$ does not depend on innovation $\left\{\xi_{n}\right\}$.

As noted in [1], the statistical estimate for the parameter $\beta$ based on observations $X_{0}, X_{1}, \ldots, X_{n}$ has the form:

$$
\theta_{n}=\frac{T_{n}}{S_{n}}
$$

where $T_{n}=\sum_{k=1}^{n} X_{k} X_{k-1}$ and $S_{n}=\sum_{k=1}^{n} X_{k-1}^{2}, n \geq 1$.

Note that the statistical estimate $\theta_{n}$ of the form (2) was obtained using the least squares method [1]. The sequences $T_{n}, S_{n}$ and $\theta_{n}, n \geq 1$ are Markov random walks and play an important role in the theory of nonlinear renewal ([2], [3]).

Limit theorems for these Markov random walks were studied in [1], [2], [4] under various

\footnotetext{
${ }^{*}$ Corresponding author.

E-mail addresses: ragimovf@rambler.ru (F.G. Rahimov), ibadovairade@yandex.ru (I.A. Ibadova), leylagulu94@gmail.com (L.V. Guliyeva). 
assumptions about the innovation $\left\{\xi_{n}\right\}$ and the parameter $\beta$ of the process $A R(1)$.

These limit theorems allow us to study a number of boundary value problems related to the intersection of a linear and nonlinear boundary by random walks $T_{n}, S_{n}$ and $\theta_{n}, n \geq 1$.

Some linear boundaries of the problem for Markov random walks are studied in [2], [3], [5], [6].

Nonlinear boundary value problems for Markov random walks have been studied very little. Some results in this area were obtained in [5], [7], [8].

Consider a family of first passage times

$$
\tau_{a}=\inf \left\{n \geq 1: T_{n} \geq f_{a}(n)\right\}
$$

of the Markov random $T_{n}=\sum_{k=1}^{n} X_{k} X_{k-1}, n \geq 1$ for the nonlinear boundary $f_{a}(t), a>0, t>0$ Assume that $\inf \{\varnothing\}=\infty$.

In [7], under certain assumptions about the nonlinear boundary $f_{a}(t)$, a strong law of large numbers and a theorem on the uniform integrability of the family $\tau_{a}, a>0$, of the form (3) were proved.

In this paper, we prove the central limit theorem for the Markov random walk $T_{n}, n \geq 1$ and family (3).

Similar problems have been studied for the case of a linear boundary $f_{a}(t)=a$ in [3], [9] and [10].

\section{Problem statement and proof of the main results}

First, we note the following known facts, which we will need further.

Regarding the process $A R(1)$, we will assume that $E \xi_{1}=0, D \xi_{1}=1, E X_{0}^{2}<\infty$ and $|\beta|<$ 1 (see. [1], [2]).

Under these conditions, it was shown in [2] (see also [1]) that for $n \rightarrow \infty$ :

$$
\begin{aligned}
& \frac{T_{n}}{n} \stackrel{n . \mathrm{H.}}{\rightarrow} \lambda=\frac{\beta}{1-\beta^{2}}, \\
& \frac{S_{n}}{n} \stackrel{\text { n.н. }}{\rightarrow} \frac{1}{1-\beta^{2}}
\end{aligned}
$$

and it was proved in [1] that the central limit theorem holds for $\theta_{n}$ :

$$
\lim _{n \rightarrow \infty} P\left(\sqrt{n}\left(\theta_{n}-\beta\right) \leq x\right)=\Phi\left(\frac{x}{\delta}\right),
$$

where $x \in R$, and $\delta=\sqrt{1-\beta^{2}}, \Phi(x)=\frac{1}{\sqrt{2 \pi}} \int_{-\infty}^{x} e^{-\frac{y^{2}}{2}} d y$.

In what follows, we will assume that $\beta \in(0,1)$.

Regarding the nonlinear boundary $f_{a}(t)$, we will assume that the following regularity conditions are satisfied:

1) For each $a$, the function $f_{a}(t)$ increases, continuously differentiable for $t>0$, and $f_{a}(1) \uparrow$ $\infty$ for $a \rightarrow \infty$.

2) For each $a$, the function $\frac{f_{a}(t)}{t}$ decreases to zero for $t \rightarrow \infty$.

3) For any function $n=n(a) \rightarrow \infty, a \rightarrow \infty$, such that $\frac{f_{a}(n)}{n} \rightarrow \lambda$ for $a \rightarrow \infty$ holds $f_{a}^{\prime}(n) \rightarrow$ $\mu \in(0, \lambda)$.

By virtue of the assumptions made, the equation $f_{a}(n)=n \lambda$ has a unique solution $N_{a}=$ $N_{a}(\lambda)$, and $N_{a} \rightarrow \infty$ for $a \rightarrow \infty$.

Note that for the family of functions $f_{a}(t)=a t^{\varepsilon}, 0<\varepsilon<1$, conditions $1-3$ are satisfied. 
In [7], it was proved that under the above conditions with respect to the process $A R(1)$ and the family of nonlinear boundaries $f_{a}(t)$,

$$
\tau_{a} \stackrel{n . \text { H. }}{\longrightarrow} \infty \text { and } \frac{\tau_{a}}{N_{a}} \stackrel{n . \text { H. }}{\longrightarrow} 1 \text { for } a \rightarrow \infty
$$

We have

Theorem 1. Suppose that $E \xi_{1}=0, D \xi_{1}=1, E X_{0}^{2}<\infty$ and $0<|\beta|<1$. Then for any $x \in R$

$$
\lim _{n \rightarrow \infty} P\left(\sqrt{n}\left(\frac{T_{n}}{n}-\lambda\right) \leq x\right)=\Phi(x \delta) .
$$

Proof. It is clear that by virtue of (3) and (4) $\theta_{n} \stackrel{n \text {.н. }}{\rightarrow} \beta$ for $n \rightarrow \infty$. Then, given that $\frac{T_{n}}{n \theta_{n}}$ $\stackrel{n . \text { н. }}{\rightarrow} \frac{1}{1-\beta^{2}}$ for $n \rightarrow \infty$, from (6) we have:

$$
\begin{gathered}
\lim _{n \rightarrow \infty} P\left(\sqrt{n}\left(\frac{T_{n}}{n}-\lambda\right) \leq x\right)=\lim _{n \rightarrow \infty} P\left(\frac{T_{n}}{n} \leq \frac{x}{\sqrt{n}}+\lambda\right)= \\
=\lim _{n \rightarrow \infty} P\left(\theta_{n} \frac{T_{n}}{n \theta_{n}} \leq \frac{x}{\sqrt{n}}+\lambda\right)=\lim _{n \rightarrow \infty} P\left(\theta_{n} \leq \frac{x\left(1-\beta^{2}\right)}{\sqrt{n}}+\lambda\left(1-\beta^{2}\right)\right)= \\
=\lim _{n \rightarrow \infty} P\left(\theta_{n} \leq \frac{x\left(1-\beta^{2}\right)}{\sqrt{n}}+\beta\right)=\lim _{n \rightarrow \infty} P\left(\sqrt{n}\left(\theta_{n}-\beta\right) \leq x\left(1-\beta^{2}\right)\right)= \\
=\Phi\left(\frac{x\left(1-\beta^{2}\right)}{\delta}\right)=\Phi(x \delta),
\end{gathered}
$$

since $\delta^{2}=\left(1-\beta^{2}\right)$.

Note 1. Note that the statement of Theorem 1 was given without proof in [2], where the value of the parameter of the limiting distribution was specified erroneously.

Using Theorem 1, we prove the following central limit theorem for the family $\tau_{a}, a>0$, of the form (3).

Theorem 2. Suppose that $E \xi_{1}=0, D \xi_{1}=1, E X_{0}^{2}<\infty$ and $0<\beta<1$ and conditions 1,2 , and 3 are satisfied. Then for $x \in R$

$$
\lim _{a \rightarrow \infty} P\left(\sqrt{N_{a}}\left(\frac{\tau_{a}}{N_{a}}-1\right) \leq x\right)=\Phi(x \gamma)
$$

where $\gamma=\delta(\lambda-\mu)$.

To prove this theorem, we need the following lemmas.

Lemma 1. Suppose that the sequence $Y_{n}, n \geq 1$ of random variables converges in distribution to the random variable $Y$, i.e., $Y_{n} \stackrel{d}{\rightarrow} Y$ for $n \rightarrow \infty$, and it is uniformly continuous in probability, i.e., it satisfies the condition

$$
\lim _{\delta \rightarrow 0} \sup _{n} P\left(\max _{1 \leq k \leq n \delta}\left|Y_{n+k}-Y_{n}\right|>\varepsilon\right)=0
$$

For any $\varepsilon>0$. such that

Furthermore, suppose that $\eta_{a}, a>0$ is a family of non-negative integer random variables

$$
\frac{\eta_{a}}{m_{a}} \stackrel{P}{\rightarrow} c>0
$$

where $m_{a} \rightarrow \infty$ for $a \rightarrow \infty$ and $c$ is a constant. 
Then $Y_{\eta_{a}} \stackrel{d}{\rightarrow} Y$ for $a \rightarrow \infty$.

The statement of this lemma follows from Anscombe's theorem proved in [11] (see also [12]).

\section{Lemma 2.}

1) If the sequence $Y_{n}, n \geq 1$ converges almost surely to a finite limit, then it is uniformly continuous in probability.

2) If the sequences $Y_{n}$ and $Z_{n}, n \geq 1$ are uniformly continuous, then their sum $Y_{n}+Z_{n}, n \geq$ 1 is also uniformly continuous in probability. Moreover, if the sequences $Y_{n}$ and $Z_{n}, n \geq 1$ are stochastically bounded, then their product $Y_{n} Z_{n}, n \geq 1$ is uniformly continuous in probability.

Statement 2 of this lemma was proved in [12], and statement 1 immediately follows from the definition of convergence almost sure ([11]).

Lemma 3. The sequence $T_{n}^{*}=\sqrt{n}\left(\frac{T_{n}}{n}-\lambda\right), n \geq 1$ is uniform in probability.

The statement of Lemma 3 is proved in [2].

Proof of Theorem 2. Denote $R_{a}=T_{\tau_{a}}-f_{a}\left(\tau_{a}\right)$ and $\tau_{a}^{*}=\frac{\tau_{a}-N_{a}}{\sqrt{\tau_{a}}}$. We have

$$
\begin{gathered}
\frac{T_{\tau_{a}}-\lambda \tau_{a}}{\sqrt{\tau_{a}}}=\frac{f_{a}\left(\tau_{a}\right)-\lambda \tau_{a}}{\sqrt{\tau_{a}}}+\frac{R_{a}}{\sqrt{\tau_{a}}}=\frac{f_{a}\left(N_{a}\right)-\lambda \tau_{a}}{\sqrt{\tau_{a}}}+\frac{R_{a}}{\sqrt{\tau_{a}}}= \\
=\frac{f_{a}\left(N_{a}\right)-\lambda \tau_{a}}{\sqrt{\tau_{a}}}+\frac{f_{a}\left(\tau_{a}\right)-f_{a}\left(N_{a}\right)}{\sqrt{\tau_{a}}}+\frac{R_{a}}{\sqrt{\tau_{a}}} .
\end{gathered}
$$

By the mean value theorem,

$$
f_{a}\left(\tau_{a}\right)-f_{a}\left(N_{a}\right)=f_{a}^{\prime}\left(v_{a}\right)\left(\tau_{a}-N_{a}\right)
$$

where $v_{a}$ is an intermediate point between $N_{a}$ and $\tau_{a}$.

Then, given that $f_{a}\left(N_{a}\right)=\lambda N_{a}$, from (9) we get

$$
\frac{T_{\tau_{a}}-\lambda \tau_{a}}{\sqrt{\tau_{a}}}=\lambda \frac{N_{a}-\tau_{a}}{\sqrt{\tau_{a}}}+f_{a}^{\prime}\left(v_{a}\right) \frac{\tau_{a}-N_{a}}{\sqrt{\tau_{a}}}+\frac{R_{a}}{\sqrt{\tau_{a}}}
$$

or

$$
\frac{T_{\tau_{a}}-\lambda \tau_{a}}{\sqrt{\tau_{a}}}=\tau_{a}^{*}\left(f_{a}^{\prime}\left(v_{a}\right)-\lambda\right)+\frac{R_{a}}{\sqrt{\tau_{a}}} .
$$

It follows from Theorem 1 and Lemmas 1 and 3 that

$$
\lim _{a \rightarrow \infty} P\left(\frac{T_{\tau_{a}}-\lambda \tau_{a}}{\sqrt{\tau_{a}}} \leq x\right)=\Phi(x \delta) .
$$

Let us prove that the second term in (10) converges to zero in probability for $a \rightarrow \infty$, i.e.,

$$
\frac{R_{a}}{\sqrt{\tau_{a}}} \stackrel{P}{\rightarrow} 0
$$

Indeed, given that $T_{\tau_{a}-1}<f_{a}\left(\tau_{a}\right)$ by definition of $\tau_{a} \mathrm{c}$

$$
0 \leq R_{a}=T_{\tau_{a}}-f_{a}\left(\tau_{a}\right)<T_{\tau_{a}}-T_{\tau_{a}-1}=\mathrm{X}_{\tau_{a}} \mathrm{X}_{\tau_{a}-1}
$$

Hence it is clear that to prove (12) it is sufficient to show that

$$
\frac{\mathrm{X}_{\tau_{a}} \mathrm{X}_{\tau_{a}-1}}{\sqrt{\tau_{a}}} \stackrel{P}{\rightarrow} 0 \text { for } a \rightarrow \infty .
$$


To this end, we first prove that

$$
\frac{\mathrm{X}_{n} \mathrm{X}_{n-1}}{\sqrt{n}} \stackrel{P}{\rightarrow} 0 \text { for } n \rightarrow \infty .
$$

Using the Chebyshev inequality and the Cauchy-Schwartz inequality, we have:

$$
P\left(\left|X_{n} X_{n-1}\right| \geq \varepsilon \sqrt{n}\right) \leq \frac{E\left|X_{n} X_{n-1}\right|}{\varepsilon \sqrt{n}} \leq \frac{1}{\varepsilon} \sqrt{\frac{E X_{n}^{2} \cdot E X_{n-1}^{2}}{n}} .
$$

It was shown in [1] that under the condition of Theorem 1,

$$
E X_{n}^{2} \rightarrow \frac{1}{1-\beta^{2}} \text { for } n \rightarrow \infty .
$$

Therefore, (15) implies (14).

Further, given that $X_{n} X_{n-1}=T_{n}-T_{n-1}$ m we have:

$$
\frac{\mathrm{X}_{n} \mathrm{X}_{n-1}}{\sqrt{n}}=\frac{T_{n}-T_{n-1}}{\sqrt{n}}=\frac{T_{n}-n \lambda}{\sqrt{n}}-\frac{T_{n-1}-n \lambda}{\sqrt{n}}=T_{n}^{*}-T_{n}^{*} \sqrt{\frac{n-1}{n}}+\frac{\lambda}{\sqrt{n}},
$$

where $T_{n}^{*}=\frac{T_{n}-n \lambda}{\sqrt{n}}$.

By virtue of Lemma 2 and Lemma 3, it follows from the last equality that the sequence $\frac{\mathrm{x}_{n} \mathrm{X}_{n-1}}{\sqrt{n}}, n \geq 1$ is uniformly continuous in probability.

Then from (14) and Lemma 1 it follows (13). Thus, we have proved convergence (12).

Now we will show that in equality (10)

$$
f^{\prime}\left(\gamma_{a}\right) \stackrel{n . \text { H. }}{\rightarrow} \mu \text { for } a \rightarrow \infty .
$$

By virtue of condition 3) with respect to the functions $f_{a}(t)$, we should show that

$$
\frac{f_{a}\left(\gamma_{a}\right)}{\gamma_{a}} \stackrel{n . \text { H. }}{\rightarrow} \lambda \text { for } a \rightarrow \infty
$$

For definiteness, assume $\tau_{a} \leq \gamma_{a} \leq N_{a}$.

Then, by virtue of condition 2 , we have

$$
\mu=\frac{f_{a}\left(N_{a}\right)}{N_{a}} \leq \frac{f_{a}\left(\gamma_{a}\right)}{\gamma_{a}} \leq \frac{f_{a}\left(\tau_{a}\right)}{\tau_{a}}
$$

Let us prove that $\frac{f_{a}\left(\tau_{a}\right)}{\tau_{a}} \stackrel{\text { n.н. }}{\rightarrow} \lambda$ for $a \rightarrow \infty$

By the definition of $\tau_{a}$, we can write

$$
\frac{T_{\tau_{a}-1}}{\tau_{a}}<\frac{f_{a}\left(\tau_{a}\right)}{\tau_{a}} \leq \frac{T_{\tau_{a}}}{\tau_{a}}
$$

As can be seen, in order to prove (19), it is necessary to show that

We denote

$$
\frac{T_{\tau_{a}}}{\tau_{a}} \stackrel{n . \text { H. }}{\rightarrow} \lambda \text { for } n \rightarrow \infty
$$

$$
\begin{aligned}
& A=\left\{\omega: \frac{T_{n}}{n} \rightarrow \lambda, n \rightarrow \infty\right\}, \\
& B=\left\{\omega: \tau_{a} \rightarrow \infty, a \rightarrow \infty\right\}
\end{aligned}
$$


and

$$
C=\left\{\omega: \frac{T_{\tau_{a}}}{\tau_{a}} \rightarrow \lambda, a \rightarrow \infty\right\}
$$

By virtue of (4), $P(A)=1$, and by virtue of (7), $P(B)=1$. It is clear that $A \cap B \subset C$. Hence, given that $P(A B)=1$, By virtue of $P(C)=1$.

Consequently, convergence (21) takes place.

Then (20) implies (19).

Therefore, from (10), (11), and (12) we have:

$$
\lim _{a \rightarrow \infty} P\left(\tau_{a}^{*}(\mu-\lambda) \leq x\right)=\Phi(x \delta)
$$

or

$$
1-\lim _{a \rightarrow \infty} P\left(\tau_{a}^{*}(\lambda-\mu) \leq-x\right)=\Phi(x \delta) .
$$

Hence, applying the equality $\Phi(x)+\Phi(-x)=1$, we have that

$$
\lim _{a \rightarrow \infty} P\left(\tau_{a}^{*}(\lambda-\mu) \leq x\right)=\Phi(x \delta)
$$

By virtue of (7) from the last equality, we complete the proof of Theorem 2.

Note 2. Note that Theorem 2 for an ordinary random walk formed by sums of independent random variables was proved in [13]. Note also that similar theorems were proved in [14] for the case when the random walk is described by the trajectories of the Markov chain.

\section{Conclusion}

In this article, we consider a family of first passage times of a Markov random walk described by a first-order autoregressive process for nonlinear boundaries. The central limit theorem has been proved for the Markov random walk. Using this theorem, we have proved the central limit theorem for the family of first passage times of this walk for nonlinear boundaries.

Acknowledgement. This work supported by the Science Development Foundation under the President of the Republic of Azerbaijan - Grant No EIF-ETL-2020-2(36)-16/05/1-M-05.

\section{References}

[1] D. Pollard, Convergence of Stochastic Processes. Springer, New-York, 1984.

[2] V.F. Melfi, Nonlinear Markov renewal theory with statistical applications, The Annals of Probability. 20 No.2 (1992) pp.753-771.

[3] F.H. Rahimov, F.D. Azizov, V.S. Khalilov, Integral limit theorem for the first passage time for the level of random walk, described with AR (1) sequences, Transaction of NAS of Azerbaijan. 32 No.4 (2012) pp.95-100.

[4] Y. Zhang, X. Yang, Limit Theory for Random Coefficient First-Order Autoregressive Process, Communications in Statistics. 39 (2010) pp.1922-1931.

[5] A.A. Novikov, Some remarks on distribution of the first passage time and optimal stop of AR (1)-sequences, Theoriya veroyatn. i ee primen. 53 No.3 (2008) pp.458-471.

[6] S.A. Aliyev, F.H. Rahimov, A.D. Farhadova, V.S. Khalilov, Limit theorems for family of first passage time of the level by random walk described by a nonlinear function of the sequence autoregressive process of order one (AR (1)), Uzbek Mathematical Journal. No.1 (2019) pp. 4-14.

[7] Ф.Г. Рагимов, Т.Е. Гашимова, Л.В. Кулиева, О равномерной интегрируемости семейства моментов первого пересечения нелинейной границы случайный блужданием, описываемом процессом авторегресии, Journal of Baku Engineering University, Mathematics and Computer Science. 3 No.2 (2019) pp. 84-90. [In Russian: F.G. Rahimov, T.Ye. Hashimova, L.V. Guliyeva, On the uniform integrability of a family of first passage times of a random walk described by an autoregressive process for nonlinear boundaries].

[8] F.H. Rahimov, I.A. Ibadova, V.S. Khalilov, Limit theorems for a family of the first passage times of a parabola by 
the sums of the squares autoregression process of order one (AR (1)), Uzbek Mathematical Journal. No.2 (2019) pp.81-88.

[9] F.H. Rahimov, V.A. Abdurakhmanov, T.E. Hashimova, On the asymptotics of the mean value of the moment of the moment of the first Level-crossing by the first order autoregression process of order one (AR(1)), Transaction of NAS of Azerbaijan. 34 No.4 (2014) pp.93-96.

[10] Kh.A. Jafarova, I.A. Ibadova, V.A. Abdurakhmanov, On asymptotic behavior of the mean value of the first passage time of the level by a random walk described by autoregression process of order one (AR(1)), Transaction of NAS of Azerbaijan. 35 No.1 (2015) pp.47-50.

[11] A. Gut, Stopped random walks. Limit theorems and applications, Springer, New York, 1988.

[12] M. Woodroofe, Nonlinear renewal theory in sequential analysis, SIAM, Philadelphia, 1982.

[13] Ф.Г. Рагимов, Интегральные предельные теоремы для времени пересечения нелинейных границ суммами независимых величин, Теория вероят. и ее примен. 50 No.1 (2005) pp.158-161. [In Russian: F.G. Rahimov, Integral limit theorems for the passage time of sums of independent quantities for nonlinear boundaries, Teoriya veroyat. i yeye primen.].

[14] Ф.Г. Рагимов, Ф.Д. Азизов, Интегральные предельные теоремы для момента времени пересечения нелинейних границ цепей Маркова, Теория вероят. и ее примен. 57 No.1 (2012) pp.178-185. [In Russian: F.G. Rahimov, F.D. Azizov, Integral limit theorems for the passage time of Markov chains for nonlinear boundaries, Teoriya veroyat. i yeye primen.]. 